\title{
Maki Parameter and Upper Critical Field of the Heavy-Fermion Superconductor $\mathbf{U B e}_{13}$
}

\author{
Yusei SHIMIZU ${ }^{1 *}$, Yoichi IKEDA ${ }^{1,2}$, Takumi WAKABAYASHI ${ }^{1}$, Yoshinori HAGA ${ }^{3}$, Kenichi TENYA ${ }^{4}$, \\ Hiroyuki HIDAKA ${ }^{1}$, Tatsuya YANAGISAWA ${ }^{5}$, and Hiroshi AMITSUKA ${ }^{1}$ \\ ${ }^{1}$ Graduate School of Science, Hokkaido University, Sapporo 060-0810, Japan. \\ ${ }^{2}$ Graduate School of Natural Science and Technology, Okayama University, Okayama 700-8530, Japan. \\ ${ }^{4}$ Advanced Science Research Center, Japan Atomic Energy Agency, Tokai 319-1195, Japan. \\ ${ }^{3}$ Faculty of Education, Shinshu University, Nagano 390-8621, Japan. \\ ${ }^{5}$ Creative Research Institution, Hokkaido University, Sapporo 001-0021, Japan.
}

\begin{abstract}
We have performed low-temperature specific-heat measurements in magnetic fields for a single crystal $\mathrm{UBe}_{13}$. It has been observed that our sample exhibits a superconducting transition at an intermediate temperature $\left(T_{\mathrm{c}} \sim 0.81 \mathrm{~K}\right)$ between previously reported values for two variant samples called $\mathrm{H}$ type and $\mathrm{L}$ type. The specific heat $C(T)$ of our sample shows a $T^{3}$ behavior below $\sim 0.7 T_{\mathrm{c}}$, which is similar to the behavior of the H-type sample, suggesting the existence of point nodes in the superconducting gap function. We have obtained the upper-critical-field curves $H_{\mathrm{c} 2}$ for the [001], [110], and [111] crystal axes, which show no anisotropy at least down to the lowest measured temperature of $0.5 \mathrm{~K}$. We have also derived the Maki parameter $\kappa_{2}$, and it has been revealed that the $\kappa_{2}$ steeply decreases isotropically upon cooling just below $T_{\mathrm{c}}$. Paramagnetic effects and the symmetry of Cooper pairing of $\mathrm{UBe}_{13}$ are discussed.
\end{abstract}

KEYWORDS: Unconventional Superconductor, Heavy Fermion, Paramagnetic Effect

The heavy-fermion compound $\mathrm{UBe}_{13}$ has attracted much attention since the discovery of unconventional superconductivity 27 years ago. ${ }^{1)} \mathrm{UBe}_{13}$ has a cubic structure with the space group $O_{h}{ }^{6}(\mathrm{Fm}-3 \mathrm{c})$, and exhibits a superconducting (SC) transition at around $0.85 \mathrm{~K}$ with a large specific-heat jump $\Delta C$. Despite prolonged scientific efforts to unravel the superconductivity of this material, many open questions remain, in particular concerning the symmetry of Cooper pairing and the unusual SC phase diagram. ${ }^{2,3)}$

As for the SC gap structure, which is related to the parity of Cooper pairing, the temperature dependence of low- $T$ specific heat $C(T)^{4)}$ and magnetic field penetration depth $\lambda(T)^{5)}$ suggest the presence of point nodes, whereas nuclear magnetic resonance (NMR) spin-relaxation rate $1 / T_{1}{ }^{6}$ ) and ultrasonic attenuation $\alpha(T)^{7)}$ suggest a line node. A $p$-wave AndersonBrinkman-Morel (ABM) state, which is identified as the A phase in superfluid ${ }^{3} \mathrm{He},{ }^{8)}$ has been proposed for the SC of $\mathrm{UBe}_{13}$ from the $T^{3}$-like behavior observed in the low- $T$ specific heat. ${ }^{4)}$ An early NMR study also suggests the occurrence of the ABM state, because the Knight shift does not decrease below $T_{\mathrm{c}} \cdot{ }^{9)}$ However, recent muon spin-rotation $(\mu \mathrm{SR})$ measurements performed in field, $H \|$ [001], have revealed that the muon Knight shift shows a clear decrease below $T_{\mathrm{c}}{ }^{10}{ }^{10}$ Furthermore, a recent NMR study by Tou et al. has suggested that the Knight shift decreases below $T^{*}\left(\sim 0.7 \mathrm{~K}<T_{\mathrm{c}}\right)$ for [001], whereas increases below $T^{*}$ for [111]. ${ }^{11)}$ Therefore, no consensus has been achieved regarding the behavior of Knight shift below $T_{\mathrm{c}}$ as well as the SC-gap structure. Namely, it is still debated whether the Cooper-pairing state of $\mathrm{UBe}_{13}$ is triplet or singlet.

In order to obtain further information about the parity of Cooper pairing, it is important to investigate the presence (or absence) of paramagnetic effects. The value of $H_{\mathrm{c} 2}$ estimated experimentally by extrapolation to $T=0$ for $\mathrm{UBe}_{13}$ is about 80-130 kOe, which is much larger than the Pauli limit

E-mail address: yusei@phys.sci.hokudai.ac.jp
$H_{\mathrm{P}}(T=0)$ of $18.4 T_{\mathrm{c}} \mathrm{kOe}\left(\sim 16.6 \mathrm{kOe}\right.$ for $\left.T_{\mathrm{c}}=0.9 \mathrm{~K}\right)$. The paramagnetic effects might be thus considered to be absent in $\mathrm{UBe}_{13}$. However, this expression of $H_{\mathrm{P}}$ is reliable only when a spin-orbit scattering by magnetic impurities can be neglected. Therefore, this large $H_{\mathrm{c} 2}$ compared with the $H_{\mathrm{P}}$ is insufficient to conclude that there is no paramagnetic effect in $\mathrm{UBe}_{13}$.

Unconventional superconductors often exhibit anisotropy of $H_{\mathrm{c} 2}(T)$, which could reflect the symmetry of SC order parameter $^{12)}$ as well as anisotropy of effective mass of electron. Aliev et al. have reported that a single crystal of $\mathrm{UBe}_{13}$ shows an anisotropy of $H_{\mathrm{c} 2}$ between [001] and [110] directions, ${ }^{13)}$ while Signore et al. have observed no anisotropy. ${ }^{14)}$ This discrepancy is probably due to a sample-quality difference; to our knowledge, however, there has been no experimental study on the anisotropy of $H_{\mathrm{c} 2}$ for $\mathrm{UBe}_{13}$, except these two reports. The further study using another single crystal will thus be useful and necessary.

In the present work, we have performed low- $T$ specific heat measurements on a single crystal of $\mathrm{UBe}_{13}$ under magnetic fields applied for three different crystal axes, and investigated the anisotropy of $H_{\mathrm{c} 2}$. We have also studied the temperature dependence and the anisotropy of the Maki parameter $\kappa_{2}$, which reflects the paramagnetic effects of Cooper pairing.

A single crystal $\mathrm{UBe}_{13}$ was grown by an Al-flux method described in ref. 15 . Its dimensions were $\sim 1.8 \times 1.9 \times 0.8$ $\mathrm{mm}^{3}$ and weight was $6.6 \mathrm{mg}$. The sample orientation was checked by X-ray Laue methods. The specific-heat measurements were performed by a standard semi-adiabatic heatpulse method in zero magnetic field and by a thermal relaxation method in magnetic fields, using ${ }^{3} \mathrm{He}$ refrigerators. The magnetic field was applied up to $4.5 \mathrm{~T}$, along the three different cubic crystal axes: [001], [110], and [111].

Figure 1 shows the temperature dependence of the zerofield specific heat of $\mathrm{UBe}_{13}$ above $0.3 \mathrm{~K}$. The peak-top temperature $T_{\mathrm{c}}^{\text {top }}$ of the specific-heat jump is estimated to be $0.79 \mathrm{~K}$. From the entropy conservation for the SC transition, on the other hand, $T_{\mathrm{c}}$ is estimated to be $\sim 0.81 \mathrm{~K}$. Recently, 


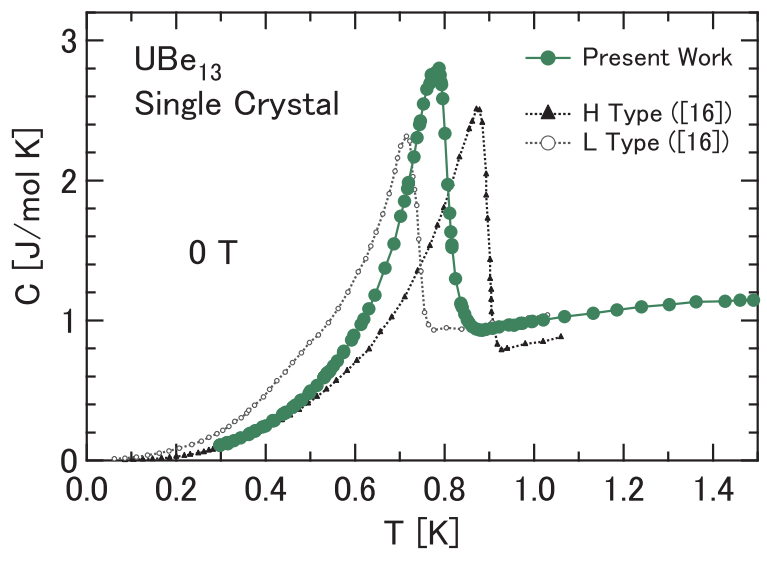

Fig. 1. The temperature dependence of the specific heat of the single crystalline $\mathrm{UBe}_{13}$ in zero field. Considering the entropy conservation at second-order transition, $T_{\mathrm{c}}$ is estimated to be $0.81 \mathrm{~K}$. Data of H-type and L-type samples from ref. 16 are also plotted for comparison.

it has been reported that there are two variants in pure $\mathrm{UBe}_{13}$, called $\mathrm{H}$ (high) and L (low) type. ${ }^{16)}$ An H-type $\mathrm{UBe}_{13}$ exhibits a SC transition at $\sim 0.85-0.9 \mathrm{~K}$, and has a broad anomaly at $T_{\mathrm{L}} \sim 0.7 \mathrm{~K}$ in thermal expansion under zero magnetic field. This anomaly cannot be seen in the temperature dependence of the specific heat at zero field, but in field, the isothermal specific heat curves $C(B)$ show a broad anomaly at a corresponding field called $B^{*}\left(T_{\mathrm{L}}\right) .{ }^{17)}$ On the other hand, an L-type $\mathrm{UBe}_{13}$ has a lower $T_{\mathrm{c}}$ of $\sim 0.75 \mathrm{~K}$, and it exhibits an obvious anomaly named $T_{\mathrm{A}}$ below $T_{\mathrm{c}}$ in the temperature dependence of the zero-field specific heat. It has been found that $T_{\mathrm{A}}$ corresponds to the thermal-expansion anomaly $T_{\mathrm{L}}{ }^{16)}$

In the present study, we could not detect any anomalies in the specific heat below $T_{\mathrm{c}}$ within the experimental accuracy. It should also be noted that the specific-heat jump $\Delta C$ at $T_{\mathrm{c}}$ of our sample is larger than those of L-type and H-type samples. It is considered that $\mathrm{UBe}_{13}$ is a strong-coupling superconductor because a dimensionless value, $\Delta C /\left.C_{\mathrm{n}}\right|_{T=T_{\mathrm{c}}}(\equiv \delta C)$ are considerably larger than 1.43 , which is expected from the weak-coupling BCS theory. The $\delta C$ value of an H-type sample is reported to be $\sim 2.6$ and a L-type sample $\sim 2.0 .{ }^{16,17)}$ on the other hand, our sample shows $\delta C$ of $2.9 \pm 0.3$, if estimated by the entropy conservation analysis, and this value is close to the value of the H-type sample. Hence, the properties seen in the specific heat of our sample is similar to those of the $\mathrm{H}$ type sample rather than the L-type sample, although the $T_{\mathrm{c}}$ is different from the values of each type.

Figure 2 shows a $C(T) / T$ versus $T^{2}$ plot of $\mathrm{UBe}_{13}$ of our experimental results in zero field. The data reported for an $\mathrm{H}$ type polycrystal in ref. 4 and an H-type single crystal in ref. 16 are also plotted. Although $T_{\mathrm{c}}$ is different, our sample and the H-type sample both reveal the $T^{3}$ power-law temperature dependence of $C(T)$ in the low temperature range $T<0.7 T_{\mathrm{c}}$. This behavior supports the proposal by Ott et al. that there are point nodes in the $\mathrm{SC}$ gap of $\mathrm{UBe}_{13}$. As mentioned above, however, the correspondence between the $C(T)$ behavior and the microscopic observation associated with the SC gap is not clear in this system, because of its delicate dependence on sample quality. Therefore, in order to establish a reliable specification of the SC gap symmetry of $\mathrm{UBe}_{13}$, it should be

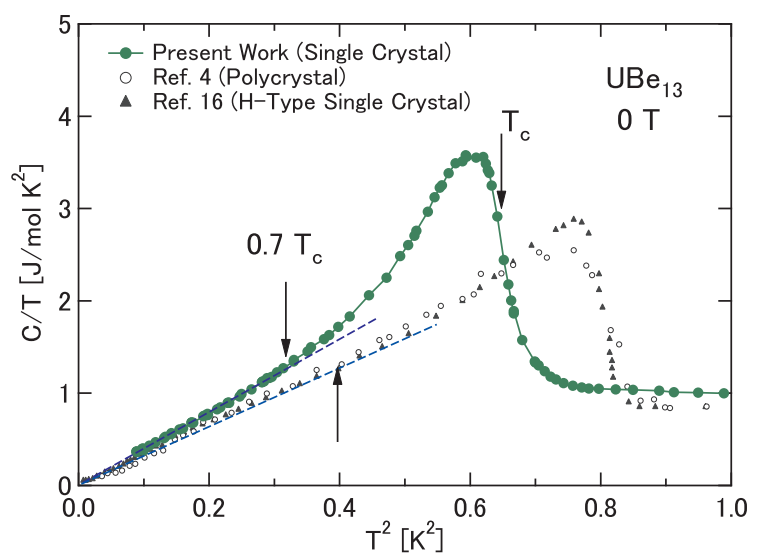

Fig. 2. $C(T) / T$ vs $T^{2}$ plot of $\mathrm{UBe}_{13}$ in zero field. Data from refs. 4 and 16 are also plotted for comparison. These $C(T)$ data roughly obey the relation $C(T) \propto T^{3}$ in the temperature range below $\sim 0.7 T_{\mathrm{c}}$, which is indicated by arrows. Broken lines are guides to the eye.

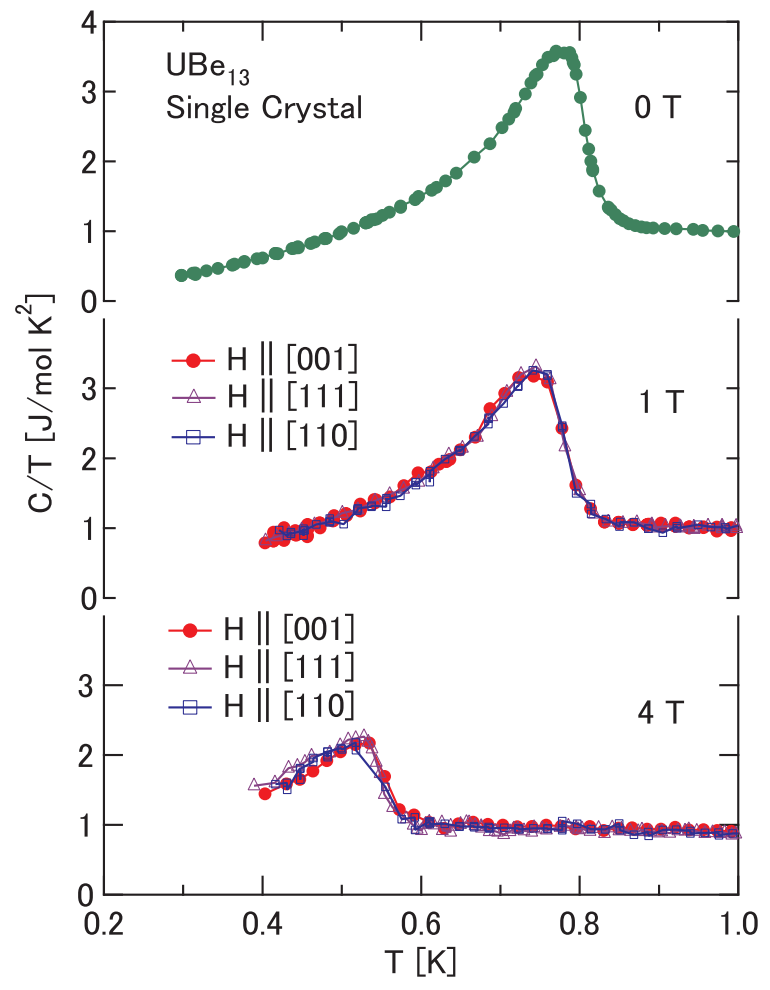

Fig. 3. $C(T) / T$ of the single crystalline $\mathrm{UBe}_{13}$ in 0,1 , and $4 \mathrm{~T}$ for three different crystal axes.

necessary to investigate the low- $T$ quasiparticle excitations by measuring various quantities on a same-quality single crystal.

Figure 3 shows the temperature dependence of the specific heat $C(T) / T$ measured at the fields 0,1 , and $4 \mathrm{~T}$ for the directions: [001], [110], and [111]. There is no significant anisotropy in the magnitude of $C(T)$ and $T_{\mathrm{c}}$ within the experimental error. Figure 4 shows the results of $H_{\mathrm{c} 2}$ for [001] and $H_{\mathrm{c} 2}^{\text {top }}$ for [001], [110], and [111], where $H_{\mathrm{c} 2}$ and $H_{\mathrm{c} 2}^{\text {top }}$ are the upper critical fields estimated from $T_{\mathrm{c}}(H)$ and $T_{\mathrm{c}}^{\mathrm{top}}(H)$, respectively. The upper critical field obtained by our previous DC magnetization measurements for the same sample ${ }^{18)}$ is also plotted as $H_{\mathrm{c} 2}^{\mathrm{mag}} . H_{\mathrm{c} 2}^{\mathrm{top}}$ is in good agreement with $H_{\mathrm{c} 2}^{\mathrm{mag}}$, while $H_{\mathrm{c} 2}$, which is obtained by considering entropy conservation at $T_{\mathrm{c}}$, is slightly larger than $H_{\mathrm{c} 2}^{\mathrm{top}}$ and $H_{\mathrm{c} 2}^{\mathrm{mag}}$. Previously, 


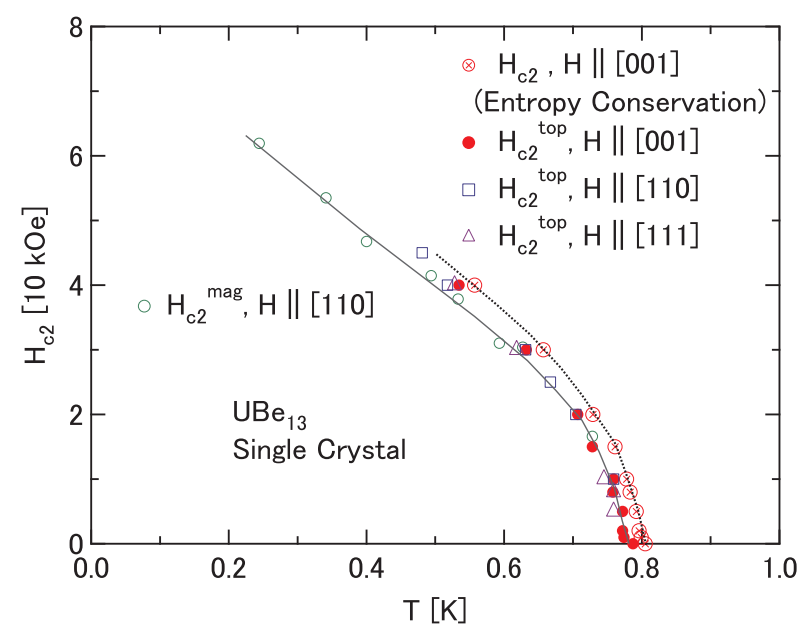

Fig. 4. The upper critical field $H_{\mathrm{c} 2}$ and $H_{\mathrm{c} 2}^{\text {top }}$ of $\mathrm{UBe}_{13}$ obtained from specific-heat measurements. As for the $H_{\mathrm{c} 2}^{\text {top }}$, the results for three different crystal axes ([001], [110], and [111]) are plotted. The solid and the dotted lines are guides to the eye. The results of previous DC magnetization measurements from ref. 18 are also plotted.

Aliev et al. have reported that $H_{\mathrm{c} 2}$ for the field direction [001] is larger than that for [110], and their difference increases to $\sim 300$ Oe at $\sim 0.7 T_{\mathrm{c}} \cdot{ }^{13)}$ Since $T_{\mathrm{c}}$ of our sample is $0.81 \mathrm{~K}$, the similar magnitude of anisotropy is expected to be observed at $\sim 0.5 \mathrm{~K}$. However, as seen in Fig. 3 and 4, the specific heat of our sample does not show any evidence for the anisotropy of $H_{\mathrm{c} 2}$, at least, down to $0.5 \mathrm{~K}$. It will be very important to investigate whether the anisotropy appears in the $H_{\mathrm{c} 2}$ curves at lower temperatures.

In general, the Maki parameter $\kappa_{2}$ can be obtained from the slope of a magnetization curve just below $H_{\mathrm{c} 2}$ by using the relation (in cgs units): $\left(d M_{\mathrm{eq}}^{\mathrm{SC}} / d H\right)_{H_{\mathrm{c} 2}}=1 / 4 \pi\left(2 \kappa_{2}^{2}-1\right) \beta_{\mathrm{A}}$, where the subscript 'eq' denotes a thermal equilibrium process, $M_{\mathrm{eq}}^{\mathrm{SC}}(H)$ a diamagnetic contribution of superconductivity, and $\beta_{\mathrm{A}}(=1.16)$ a geometric constant for triangular vortex lattice. ${ }^{19,20)}$ On the other hand, Ehrenfest's relation for a secondorder phase transition relates the slope of magnetization curve near $H_{\mathrm{c} 2}$ to the specific-heat jump $\Delta C$ as follows: ${ }^{20,21)}$

$$
\left(\frac{\Delta C}{T}\right)_{T_{\mathrm{c}}(H)}=\left(\frac{d H_{\mathrm{c} 2}}{d T}\right)_{T_{\mathrm{c}}(H)}^{2}\left(\frac{d M_{\mathrm{eq}}^{\mathrm{SC}}}{d H}\right)_{H_{\mathrm{c} 2}} .
$$

Consequently, one can obtain $\kappa_{2}$ also from the observed values of $d H_{\mathrm{c} 2} / d T$ and $\Delta C$ at $T_{\mathrm{c}}(H)$ in magnetic fields as follows:

$$
\left(\frac{\Delta C}{T}\right)_{T_{\mathrm{c}}(H)}=\left(\frac{d H_{\mathrm{c} 2}}{d T}\right)^{2} \frac{1}{4 \pi\left(2 \kappa_{2}^{2}-1\right) \beta_{A}} .
$$

In order to perform this latter procedure, we converted the specific heat per mole $[\mathrm{J} / \mathrm{mol} \mathrm{K}]$ to the value per unit volume $\left[\mathrm{erg} / \mathrm{cm}^{3} \mathrm{~K}\right]$ by using the density and molecular mass of $\mathrm{UBe}_{13}: d=4.36 \mathrm{~g} / \mathrm{cm}^{3}$ and $M=355.187$, respectively.

Figure 5 (a), (b), and (c) show the obtained temperature dependence of $\Delta C / T, d H_{\mathrm{c} 2} / d T$, and $\kappa_{2}$, respectively, for $H$ \| [001]. $\kappa_{2}$ of our sample decreases steeply with decreasing temperature below $T_{\mathrm{c}}$, and becomes constant below $\sim 0.88$ $T_{\mathrm{c}}$. According to Eq. (2), $\kappa_{2}$ is almost inversely proportional to $(\Delta C / T)^{1 / 2}$, and directly proportional to $\left|d H_{\mathrm{c} 2} / d T\right|$ in the case of $\kappa_{2}>>1$. The decrease in $\Delta C / T$ thus contributes to in-

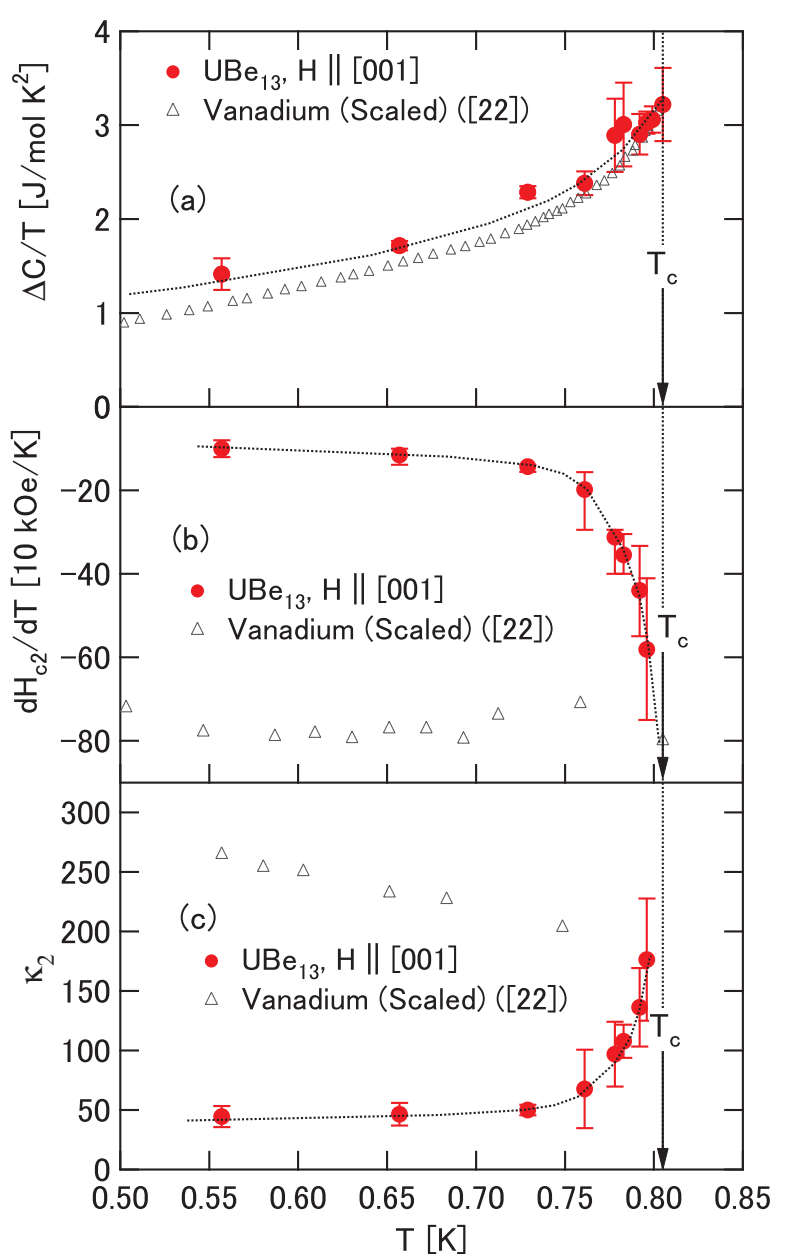

Fig. 5. The temperature dependence of (a) $\Delta C$, (b) $d H_{\mathrm{c} 2} / d T$, and (c) $\kappa_{2}$ for $H \|[001]$ of $\mathrm{UBe}_{13}$. The dotted lines are guides to the eye. The experimental results of vanadium, ${ }^{22)}$ scaled to the magnitudes of $\mathrm{UBe}_{13}$ at zero field, are also plotted for comparison.

creasing $\kappa_{2}$, while that in $\left|d H_{\mathrm{c} 2} / d T\right|$ reduces $\kappa_{2}$. In the present case, as temperature is lowered from $T_{\mathrm{c}}$ to $\sim 0.5 \mathrm{~K},\left|d H_{\mathrm{c} 2} / d T\right|$ decreases to $\sim 16 \%$, whereas $(\Delta C / T)^{1 / 2}$ decreases to $\sim 66$ $\%$. The observed reduction of $\kappa_{2}$ with the non-linear $T$ variation in $\mathrm{UBe}_{13}$ can thus mainly be attributed to the behavior of $d H_{\mathrm{c} 2} / d T$. Since there is no anisotropy in $\Delta C$ and $H_{\mathrm{c} 2}, \kappa_{2}(T)$ shows no anisotropy as well.

For the sake of comparison, we also plot in Fig. 5 the results of a type-II superconductor, vanadium $(\mathrm{V})$, quoted from ref. 22. The magnitude of $T_{\mathrm{c}}, \Delta C /\left.T\right|_{T=T_{\mathrm{c}}}, d H_{\mathrm{c} 2} /\left.d T\right|_{T=T_{\mathrm{c}}}$, and $\kappa_{2}\left(T_{\mathrm{c}}\right)\left(=\kappa_{\mathrm{GL}} \equiv \lambda / \xi\right)$ of $\mathrm{V}$ are scaled to those of $\mathrm{UBe}_{13}$ at zero field, where $\lambda$ and $\xi$ denote magnetic penetration depth and correlation length, respectively. The relative changes of $\Delta C / T$ of $\mathrm{V}$ is similar to that of $\mathrm{UBe}_{13}$, while $d H_{\mathrm{c} 2} / d T$ of $\mathrm{V}$ is nearly independent of temperature, providing a remarkable contrast with the behavior of $\mathrm{UBe}_{13}$. As a result, $\kappa_{2}$ of $\mathrm{V}$ just shows a monotonous increase upon cooling. From this comparison, we strongly suggest the peculiarity of the field distraction process of the $\mathrm{SC}$ state in $\mathrm{UBe}_{13}$; i. e., a strong bending effect of the $H_{\mathrm{c} 2}$ curve appears to be present at $\sim 0.9 T_{\mathrm{c}}$. We should also note that the absolute value of $\kappa_{\mathrm{GL}}$ for $\mathrm{UBe}_{13}$ is roughly of order $10^{2}$, which is two orders of magnitude larger than that of $\mathrm{V}(\sim 0.85)$.

The decrease of $\kappa_{2}$ upon cooling could reflect a param- 
agnetic effect. Generally, energy due to spin susceptibility $\chi_{\mathrm{s}} H^{2} / 2$ of a heavy-fermion system is large and comparable with the condensation energy of SC state: $\chi_{\mathrm{s}} H^{2} / 2 \sim H_{\mathrm{c}}^{2} / 8 \pi$. In this case, when two antiparallel-spin electrons form a Cooper pair, the electrons could change from the SC state to the spin-polarized normal state in a high field $\left(\sim H_{\mathrm{P}}\right)$ to gain the magnetic energy of the system. Consequently, the $H_{\mathrm{c} 2}(T)$ in high-field region is suppressed (paramagnetic effect). ${ }^{23)}$ Since the slope of magnetization curve $d M_{\mathrm{eq}}^{\mathrm{SC}}(H) / d H$ near $H_{\mathrm{c} 2}$ becomes larger at low temperatures, $\kappa_{2}(T)$ decreases upon cooling. In the case of spin-singlet pairing, the paramagnetic effect should be present for all directions, and thus $\kappa_{2}(T)$ isotropically decreases by cooling in fields. For example, the heavy-fermion superconductor $\mathrm{CeCoIn}_{5}$, which is considered to have an even-parity pairing, reveals a clear decrease of $\kappa_{2}$ regardless of the field direction. ${ }^{24)}$ In the case of spin-triplet pairing, on the other hand, if spin-orbit coupling is negligibly small, the paramagnetic effect should be absent for all directions, since the total spin of Cooper pair can follow the field direction without conflicting with the SC condensation. In this case, the $\kappa_{2}(T)$ in general does not decrease but increases upon cooling. However, if the spin-orbit coupling is not negligible, it may lead to the paramagnetic effect: if a projection of a total spin of a Cooper pair perpendicular to the magnetic field is not zero, and locked on one direction of a crystal axis with strong spin-orbit coupling, the paramagnetic effect should be present.

Our experimental results suggest that the paramagnetic effect isotropically occurs in $\mathrm{UBe}_{13}$, and this does not conflict with the behavior expected for the spin-singlet pairing. An odd-parity pairing, however, might also be possible, if it leads to some isotropic paramagnetic effect at least down to $0.5 \mathrm{~K}$ $\left(\sim 0.6 T_{\mathrm{c}}\right)$. Such an isotropic paramagnetic effect may occur in a triplet state, with strong spin-orbit coupling, which belongs to a one-dimensional representation of the cubic group: the SC gap function of this state is constant over the Fermi surface, and is similar to Balian-Werthamer state of ${ }^{3} \mathrm{He}$ superfluidity. However, this one-dimensional triplet SC state cannot explain the existence of point nodes in the SC gap of this material. As for another possible explanation for the decrease in $\kappa_{2}(T)$ of $\mathrm{UBe}_{13}$, we wish to point out the effect of spinorbit scattering by magnetic impurities. Maki has numerically shown that $\kappa_{2}$ of a type-II superconductor could decrease upon cooling in the presence of strong spin-orbit scattering in a dirty limit. ${ }^{25)}$ If there are $5 f$ electrons that remain the normal state even in the SC phase, they may act as the magnetic impurities forming a scattering potential for the SC electrons. Our present data do not rule out the possibility that such spin-orbit scattering occurs frequently in $\mathrm{UBe}_{13}$. In order to elucidate the origin of the decrease in the $\kappa_{2}(T)$ of this system, it will be very crucial to investigate the temperature dependence and the anisotropy of the $\kappa_{2}$ at lower temperatures. For this purpose, DC magnetization measurements below $0.5 \mathrm{~K}$ are now in progress.

In conclusion, we have performed the low- $T$ specific heat measurements in magnetic fields on a single crystal of $\mathrm{UBe}_{13}$. The specific heat $C(T)$ of our sample obeys $T^{3}$ dependence in the temperature range of $T<0.7 T_{\mathrm{c}}$, which is similar to the behavior of the H-type samples, ${ }^{4)}$ and suggests the presence of point nodes in the SC gap of this material. We have also derived the Maki parameter $\kappa_{2}(T)$ as well as the upper critical field $H_{\mathrm{c} 2}$ down to $0.5 \mathrm{~K}$ for the three directions of [001], [110], and [111]. No anisotropy has been observed in the $\kappa_{2}(T)$ and $H_{\mathrm{c} 2}(T)$ curves within the experimental accuracy. We have shown that the $\kappa_{2}$ reveals a clear decrease upon cooling for all the directions. The experimental results suggest the presence of paramagnetic effect, which is isotropic to the investigated crystal axes, or of the effect of strong spin-orbit scattering due to magnetic impurities in $\mathrm{UBe}_{13}$, both at least, down to $0.5 \mathrm{~K}$. In order to obtain further understanding of this unusual SC state, it is needed to investigate the behavior of $\kappa_{2}$ and $H_{\mathrm{c} 2}$ at lower temperatures using the same sample.

We thank H. Tou, H. Harima, and H. Kusunose for valuable discussions. The present work was supported by Grantin-Aid for Scientific Research on Innovative Areas 'Heavy Electrons' (20102002) and Scientific Research B (19340086), and S (20224015) from the Ministry of Education, Culture, Sports, Science and Technology, Japan.

1) H. R. Ott, H. Rudigier, Z. Fisk, and J. L. Smith: Phys. Rev. Lett. 50 (1983) 1595 .

2) U. Rauchschwalbe, U. Ahlheim, F. Steglich, D. Rainer, and J. J. M. France: Z. Phys. B 60 (1985) 379.

3) M. B. Maple, J. W. Chen, S. E. Lambert, Z. Fisk, J. L. Smith, H. R. Ott, J. S. Brooks, and M. J. Naughton: Phys. Rev. Lett. 54 (1985) 477.

4) H. R. Ott, H. Rudigier, T. M. Rice, K. Ueda, Z. Fisk, and J. L. Smith: Phys. Rev. Lett. 52 (1984) 1915.

5) D. Einzel, P. J. Hirschfeld, F. Gross, B. S. Chandrasekhar, K. Andres, H R. Ott, J. Beuers, Z. Fisk, and J. L. Smith: Phys. Rev. Lett. 56 (1986) 2513.

6) D. E. MacLaughlin, C. Tien, W. G. Clark, M. D. Lan, Z. Fisk, J. L. Smith, and H. R. Ott: Phys. Rev. Lett. 53 (1984) 1833.

7) B. Golding, D. J. Bishop, B. Batlogg, W. H. Haemmerle, Z. Fisk, J. L. Smith, and H. R. Ott: Phys. Rev. Lett. 55 (1985) 2479.

8) A. J. Leggett: Rev. Mod. Phys. 47 (1975) 331.

9) C. Tien, and I. M. Jiang: Phys. Rev. B 40 (1989) 229.

10) J. E. Sonier, R. H. Heffner, G. D. Morris, D. E. MacLaughlin, O. O. Bernal, J. Cooley, J. L. Smith, and J. D. Thompson: Physica B 326 (2003) 414.

11) H. Tou: private communications

12) L. P. Gor'kov: Pis'ma Zh. Eksp. Teor. Fiz. 40, (1984) 351 [JETP Lett., 40 (1984) 1155].

13) F. G. Aliev, V. Kovachik, V. V. Moshchalkov, V. V. Pryadun, N. E. Alekseevskii, A. V. Mitin, N. Agrait, S. Vieira, and R. Villar: J. Low. Temp. Phys. 85 (1991) 359.

14) P. J. C. Signore, B. Andraka, G. R. Stewart, and M. W. Meisel: Phys. Rev. B 52 (1995) 10315.

15) Y. Haga, E. Yamamoto, T. Honma, A. Nakamura, M. Hedo, S. Araki, H. Ohkuni, and Y. Ōnuki: Physica B 259-261 (1999) 627.

16) C. Langhammer, R. Helfrich, A. Bach, F. Kromer, M. Lang, T. Michels, M. Deppe, F. Steglich, and G. R. Stewart: J. Mag. Mag. Mat. 177-181 (1998) 443.

17) F. Kromer, R. Helfrich, M. Lang, F. Steglich, C. Langhammer, A. Bach, T. Michels, J. S. Kim, and G. R. Stewart: Phys. Rev. Lett. 81 (1998) 4476.

18) Y. Shimizu, Y. Ikeda, T. Wakabayashi, K. Tenya, Y. Haga, H. Hidaka, T. Yanagisawa, and H. Amitsuka: J. Phys.: Conf. Ser. 273 (2011) 012084.

19) B. Serin: 'Type II Superconductivity: Experiments' in Superconductivity, edited by R. D. Parks (Marcel Dekker, New York, 1969), Vol. 2, Ch. 15.

20) D. Saint-James, G. Sarma, and E. J. Thomas: 'Type-II S uperconductivity' (Pergamon Press, Oxford, 1969) Ch. 7.

21) B. B. Goodman: Phys. Letters. 1 (1962) 215.

22) R. Radebaugh, and P. H. Keesom: Phys. Rev. 149 (1966) 217.

23) N. R. Werthamer, E. Helfand, and P. C. Hohenberg: Phys. Rev. 147 (1966) 295.

24) S. Ikeda, H. Shishido, M. Nakashima, R. Settai, D. Aoki, Y. Haga, H. Harima, Y. Aoki, T. Namiki, H. Sato, and Y. Ōnuki: J. Phys. Soc. Jpn. 70 (2001) 2248

25) K. Maki: Phys. Rev. 148 (1966) 362 